\title{
Morphological and Phylogenetic Analyses Reveal Two New Species of Sporocadaceae From Hainan, China
}

\author{
Zhaoxue Zhang \\ Shandong Agricultural University \\ Taichang Mu \\ Shandong Agricultural University \\ Shubin Liu \\ Shandong Agricultural University \\ Rongyu Liu \\ Shandong Agricultural University \\ Xiuguo Zhang \\ Shandong Agricultural University \\ Jiwen Xia ( $\square$ xiajiwen1@126.com) \\ Shandong Agricultural University https://orcid.org/0000-0002-7436-7249
}

\section{Research Article}

Keywords: Sporocadaceae, Monochaetia, Neopestalotiopsis, Pestalotiopsis, multigene phylogeny

Posted Date: November 11th, 2021

DOl: https://doi.org/10.21203/rs.3.rs-960719/v1

License: (c) (i) This work is licensed under a Creative Commons Attribution 4.0 International License. Read Full License

Version of Record: A version of this preprint was published at MycoKeys on April 14th, 2022. See the published version at https://doi.org/10.3897/mycokeys.88.82229. 


\section{Abstract}

Species of Sporocadaceae have often been reported as plant pathogens, endophytes or saprobic, commonly isolated from a wide range of plant hosts. The isolated fungi were studied through a complete examination based on multi-locus phylogeny of a combined dataset of ITS/ TUB2/ TEF1- , in conjunction with morphological characteristics. Nine strains isolated from Schima superba, Ficus microcarpa and Ilex chinensis in Hainan Province, China, represented four species, viz, Monochaetia schimae sp. nov., Neopestalotiopsis haikouensis sp. nov., Neopestalotiopsis piceana and Pestalotiopsis licualacola.

\section{Introduction}

Xylariales, a group of fungi distributed worldwide, whose members show considerable variability in a number of characteristics, including position of ascomata, presence and type of sterile tissues, and habit (Barr 1990). The Sporocadaceae (type genus: Sporocadus Corda) is a well-defined family in the Xylariales (Liu et al. 2019). Based on phylogenetic analyses and morphological comparison, Sporocadaceae has delimited thirty genera including Monochaetia (Sacc.) Allesch., Neopestalotiopsis Maharachch. et al., Pestalotiopsis Steyaert, Pseudopestalotiopsis Maharachch. et al., etc., which is generally congruent with the classification system proposed by Nag Raj (1993) prior to the DNA phylogeny era. Species of Sporocadaceae are endophytic, plant pathogenic or saprobic, and associated with a wide range of host plants.

Initially, pestalotia-like asexual morphs were classified in Amphisphaeriaceae by Samuels et al. (1987), accommodating 36 genera (Hawksworth et al. 1995). Its ordinal level of classification, the Amphisphaeriales, was introduced by Eriksson \& Hawksworth (1986), but treated as a synonym of Xylariales one year later by Eriksson \& Hawksworth (1987). Hawksworth et al. (1995) followed and supported this classification by molecular data. The order was recently resurrected to include Amphisphaeriaceae, Clypeosphaeriaceae and another four novel families derived from Amphisphaeriaceae, however, the sequence dataset was largely incomplete and some of the introduced families were not well supported statistically (Senanayake et al. 2015). Subsequently, Bartaliniaceae, Discosiaceae, Pestalotiopsidaceae and Robillardaceae (Crous et al. 2015) were synonymized and revived the older family name Sporocadaceae to accommodate them, together with the Amphisphaeriaceae and Phlogicylindriaceae, Sporocadaceae was accommodated in the Xylariales, however, Amphisphaeriales was not accepted due to a lack of phylogenetic support in their analysis (Jaklitsch et al. 2016).

Presently, agreement on the classification and delimitation of the family itself seems to have been reached after intense debate. Fungi in the Sporocadaceae (e.g. Bartalinia, Pestalotia, Pestalotiopsis, Robillarda, Seimatosporium, Seiridium and Truncatella) possess common asexual morphological characters related to their acervular conidiomata, conidiogenesis and conidia (Liu et al. 2019). To date, most phylogenetic studies addressing genera of Sporocadaceae have been based solely on ITS and LSU sequences (Barber et al. 2011; Tanaka et al. 2011; Jaklitsch et al. 2016), or on concatenated datasets of more genes but with incomplete datasets (Senanayake et al. 2015; Wijayawardene et al. 2016). Consequently, the taxonomic concept of, and generic delimitation within Sporocadaceae remain unclear.

Most of the genera of Sporocadaceae contain over-lapping morphological characters of conidia such as the number of median cells, colour of median cells, presence of apical and basal appendages (Jeewon et al. 2002). In this study, we made a collection of the established genera Pestalotiopsis, Neopestalotiopsis and Monochaetia species on leaves of Schima superba, Ficus microcarpa and Ilex chinensis in Hainan Province, China and the new species is established with descriptions, illustrations and molecular data based on ITS, TUB2 and TEF1- $a$ loci.

\section{Materials And Methods}

Specimen collection and morphological descriptions

The samples were collected from Hainan Province, China. The strains were isolated from diseased leaves of Schima superba, Ficus microcarpa and Ilex chinensis using tissue isolation from surface sterilized leaf tissues were conducted following the protocol of Gao et al. (2014). Tissue fragments $(0.5 \times 0.5 \mathrm{~cm})$ were taken from the margin of leaf lesions and surface-sterilized by consecutively immersing in $75 \%$ ethanol solution for $30 \mathrm{~s}, 5 \%$ sodium hypochlorite solution for $1 \mathrm{~min}$, and then rinsing in sterile 
distilled water for $30 \mathrm{~s}$. The pieces were dried with sterilized paper towels and placed on potato dextrose agar (PDA). All the plates were incubated at biochemical incubator at $25^{\circ} \mathrm{C}$ for 3-4 days, then hyphae were picked out of the periphery of the colonies and

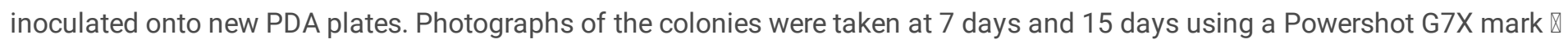
digital camera. Micromorphological characters were observed using Olympus SZX10 stereomicroscope and Olympus BX53 microscope, all fitted with Olympus DP80 high definition colour digital cameras to photo-document fungal structures. All fungal strains were stored in $10 \%$ sterilized glycerin at $4{ }^{\circ} \mathrm{C}$ for further studies. The holotype specimens are deposited in the Herbarium of Plant Pathology, Shandong Agricultural University (HSAUP). Ex-type cultures are deposited in the Shandong Agricultural University Culture Collection (SAUCC). Taxonomic information of the new taxa was submitted to MycoBank (http://www.mycobank.org).

DNA extraction, PCR amplification, and DNA sequencing

Genomic DNA was extracted from fungal mycelium on PDA, using a modified cetyltrimethylammonium bromide (CTAB) protocol as described in Guo et al. (2000). The internal transcribed spacer regions with intervening 5.8S nrRNA gene (ITS), part of the betatubulin gene region (TUB2), and partial translation elongation factor 1-alpha (TEF1- $a$ ) genes were amplified and sequenced by using primers pairs ITS4/ITS5 (White et al. 1990), T1/Bt2b (O’Donnell \& Cigelnik 1997; Glass \& Donaldson 1995), EF1-728F/EF2 (Carbone \& Kohn 1999; O’Donnell et al. 1998), respectively.

PCR was performed using an Eppendorf Master Thermocycler (Hamburg, Germany). Amplification reactions were performed in a $50 \mu \mathrm{L}$ reaction volume, which contained $25 \mu \mathrm{L}$ Green Taq Mix (Vazyme, Nanjing, China), $2 \mu \mathrm{L}$ of each forward and reverse primer $(10 \mu \mathrm{M})$ (Tsingke, Beijing, China), and $2 \mu \mathrm{L}$ template genomic DNA in amplifier, and were adjusted with distilled deionized water to a total volume of $50 \mu \mathrm{L}$. PCR parameters were as follows: $94^{\circ} \mathrm{C}$ for $5 \mathrm{~min}$, followed by 35 cycles of denaturation at $94^{\circ} \mathrm{C}$ for $30 \mathrm{~s}$, annealing at a suitable temperature for $30 \mathrm{~s}$, extension at $72^{\circ} \mathrm{C}$ for $1 \mathrm{~min}$ and a final elongation step at $72^{\circ} \mathrm{C}$ for 7 min. Annealing temperature for each gene were $55^{\circ} \mathrm{C}$ for ITS, $54^{\circ} \mathrm{C}$ for $T U B 2,52^{\circ} \mathrm{C}$ for TEF1- $a$. The PCR products were visualised on $1 \%$ agarose electrophoresis gel. Sequencing was done bi-directionally, conducted by the Tsingke Biotechnology Company Limited (Shanghai, China). Consensus sequences were obtained using MEGA 7.0 or MEGA-X (Kumar et al. 2016). All sequences generated in this study were deposited in GenBank (Table 1). 
Table 1

Species and GenBank accession numbers of DNA sequences used in this study. New sequences in bold.

\begin{tabular}{|c|c|c|c|c|c|c|}
\hline \multirow[t]{2}{*}{ Species } & \multirow[t]{2}{*}{ Strain } & \multirow[t]{2}{*}{ Host/substrate } & \multirow[t]{2}{*}{ Country } & \multicolumn{3}{|c|}{ GenBank accession number } \\
\hline & & & & ITS & TUB2 & $T E F 1$ \\
\hline $\begin{array}{l}\text { Bartalinia } \\
\text { robillardoides }\end{array}$ & CBS 122705 T & $\begin{array}{l}\text { Leptoglossus } \\
\text { occidentalis }\end{array}$ & Italy & LT853104 & LT853252 & LT853202 \\
\hline \multirow[t]{2}{*}{$\begin{array}{l}\text { Ciliochorella } \\
\text { phanericola }\end{array}$} & MFLUCC 14-0984 T & $\begin{array}{l}\text { Phanera } \\
\text { purpurea }\end{array}$ & Thailand & KX789680 & KX789682 & - \\
\hline & MFLUCC 12-0310 & $\begin{array}{l}\text { Phanera } \\
\text { purpurea }\end{array}$ & Thailand & KF827444 & KF827478 & KF827477 \\
\hline \multirow[t]{2}{*}{$\begin{array}{l}\text { Monochaetia } \\
\text { castaneae }\end{array}$} & $\begin{array}{l}\text { CFCC } 54354 \text { = SM9- } \\
1 \mathrm{~T}\end{array}$ & Castanea sp. & China & MW166222 & MW218515 & MW199741 \\
\hline & SM9-2 & Castanea sp. & China & MW166223 & MW218516 & MW199742 \\
\hline M. dimorphospora & NBRC 9980 & $\begin{array}{l}\text { Castanea } \\
\text { pubinervis }\end{array}$ & Japan & LC146750 & - & - \\
\hline \multirow[t]{2}{*}{ M. ilexae } & KUMCC 15-0520 T & Ilex sp. & China & KX984153 & - & - \\
\hline & CBS 101009 & Air & Japan & MH553953 & MH554612 & MH554371 \\
\hline M. junipericola & CBS 143391 T & $\begin{array}{l}\text { Juniperus } \\
\text { communis, } \\
\text { twig }\end{array}$ & Germany & MH107900 & MH108045 & MH108021 \\
\hline \multirow[t]{2}{*}{ M. kansensis } & PSHI2004Endo1030 & $\begin{array}{l}\text { Cyclobalaopsis } \\
\text { myrsinaefolia }\end{array}$ & China & DQ534044 & DQ534047 & - \\
\hline & PSHI2004Endo1031 & $\begin{array}{l}\text { Cyclobalaopsis } \\
\text { myrsinaefolia }\end{array}$ & China & DQ534045 & DQ534048 & - \\
\hline \multirow[t]{3}{*}{ M. monochaeta } & CBS 546.80 & $\begin{array}{l}\text { Culture } \\
\text { contaminant }\end{array}$ & Netherlands & MH554056 & MH554732 & MH554491 \\
\hline & CBS $199.82 \mathrm{~T}$ & $\begin{array}{l}\text { Quercus } \\
\text { pubescens }\end{array}$ & Italy & MH554018 & MH554694 & - \\
\hline & CBS 115004 & Quercus robur & Netherlands & AY853243 & MH554639 & MH554398 \\
\hline M. quercus & CBS $144034 \mathrm{~T}$ & $\begin{array}{l}\text { Quercus } \\
\text { eduardi }\end{array}$ & Mexico & MH554171 & MH554844 & MH554606 \\
\hline \multirow[t]{3}{*}{ M. schimae } & SAUCC212201 T & $\begin{array}{l}\text { Schima } \\
\text { superba }\end{array}$ & China & MZ577565 & OK104867 & OK104874 \\
\hline & SAUCC212202 & $\begin{array}{l}\text { Schima } \\
\text { superba }\end{array}$ & China & MZ577566 & OK104868 & OK104875 \\
\hline & SAUCC212203 & $\begin{array}{l}\text { Schima } \\
\text { superba }\end{array}$ & China & MZ577567 & OK104869 & OK104876 \\
\hline M. sinensis & HKAS 10065 T & Quercus sp. & China & MH115995 & MH115999 & - \\
\hline $\begin{array}{l}\text { Neopestalotiopsis } \\
\text { acrostichi }\end{array}$ & MFLUCC 17-1754 T & $\begin{array}{l}\text { Acrostichum } \\
\text { aureum }\end{array}$ & Thailand & MK764272 & MK764338 & MK764316 \\
\hline N. aotearoa & CBS $367.54 \mathrm{~T}$ & Canvas & $\begin{array}{l}\text { New } \\
\text { Zealand }\end{array}$ & KM199369 & KM199454 & KM199526 \\
\hline N. asiatica & $\begin{array}{l}\text { CFCC } 54339= \\
\text { SM32 }\end{array}$ & $\begin{array}{l}\text { Castanea } \\
\text { mollissima }\end{array}$ & China & MW166224 & MW218517 & MW199743 \\
\hline
\end{tabular}

Isolates marked with "T" are ex-type or ex-epitype strains. 


\begin{tabular}{|c|c|c|c|c|c|c|}
\hline \multirow[t]{2}{*}{ Species } & \multirow[t]{2}{*}{ Strain } & \multirow[t]{2}{*}{ Host/substrate } & \multirow[t]{2}{*}{ Country } & \multicolumn{3}{|c|}{ GenBank accession number } \\
\hline & & & & ITS & TUB2 & TEF1 \\
\hline & SM7 & $\begin{array}{l}\text { Castanea } \\
\text { mollissima }\end{array}$ & China & MW166225 & MW218518 & MW199744 \\
\hline N. brachiata & MFLUCC 17-1555 T & $\begin{array}{l}\text { Rhizophora } \\
\text { apiculata }\end{array}$ & Thailand & MK764274 & MK764340 & MK764318 \\
\hline \multirow[t]{3}{*}{ N. brasiliensis } & COAD $2166 \mathrm{~T}$ & $\begin{array}{l}\text { Psidium } \\
\text { guajava }\end{array}$ & Brazil & MG686469 & MG692400 & MG692402 \\
\hline & CFCC 54341 = ZY4 & $\begin{array}{l}\text { Castanea } \\
\text { mollissima }\end{array}$ & China & MW166229 & MW218522 & MW199748 \\
\hline & ZY4-2D & $\begin{array}{l}\text { Castanea } \\
\text { mollissima }\end{array}$ & China & MW166230 & MW218523 & MW199749 \\
\hline N. cubana & CBS $600.96 \mathrm{~T}$ & leaf litter & Cuba & KM199347 & KM199438 & KM199521 \\
\hline N. egyptiaca & CBS $140162 \mathrm{~T}$ & $\begin{array}{l}\text { Mangifera } \\
\text { indica }\end{array}$ & Egypt & KP943747 & KP943746 & KP943748 \\
\hline N. eucalypticola & CBS 264.37 T & $\begin{array}{l}\text { Eucalyptus } \\
\text { globulus }\end{array}$ & - & KM199376 & KM199431 & KM199551 \\
\hline \multirow[t]{2}{*}{ N. formicarum } & CBS $362.72 \mathrm{~T}$ & dead ant & Ghana & KM199358 & KM199455 & KM199517 \\
\hline & CBS 115.83 & Plant debris & Cuba & KM199344 & KM199444 & KM199519 \\
\hline \multirow[t]{2}{*}{ N. haikouensis } & SAUCC212271 T & Ilex chinensis & China & OK087294 & OK104870 & OK104877 \\
\hline & SAUCC212272 & Ilex chinensis & China & OK087295 & OK104871 & OK104878 \\
\hline N. honoluluana & CBS $114495 \mathrm{~T}$ & Telopea sp. & USA & KM199364 & KM199457 & KM199548 \\
\hline N. iraniensis & CBS $137768 \mathrm{~T}$ & $\begin{array}{l}\text { Fragaria } \\
\text { ananassa }\end{array}$ & Iran & KM074048 & KM074057 & KM074051 \\
\hline N. javaensis & CBS 257.31 T & Cocos nucifera & Indonesia & KM199357 & KM199437 & KM199543 \\
\hline N. mesopotamica & CBS 336.86 T & Pinus brutia & Iraq & KM199362 & KM199441 & KM199555 \\
\hline N. musae & MFLUCC 15-0776 T & Musa sp. & Thailand & KX789683 & KX789686 & KX789685 \\
\hline N. natalensis & CBS $138.41 \mathrm{~T}$ & $\begin{array}{l}\text { Acacia } \\
\text { mollissima }\end{array}$ & $\begin{array}{l}\text { South } \\
\text { Africa }\end{array}$ & KM199377 & KM199466 & KM199552 \\
\hline N. pandanicola & KUMCC 17-0175 T & Pandanaceae & China & - & MH412720 & MH388389 \\
\hline N. petila & MFLUCC 17-1738 T & $\begin{array}{l}\text { Rhizophora } \\
\text { mucronata }\end{array}$ & Thailand & MK764276 & MK764342 & MK764320 \\
\hline N. phangngaensis & MFLUCC 18-0119 T & Pandanaceae & Thailand & MH388354 & MH412721 & MH388390 \\
\hline \multirow[t]{4}{*}{ N. piceana } & CBS $394.48 \mathrm{~T}$ & Picea sp. & UK & KM199368 & KM199453 & KM199527 \\
\hline & CBS 254.32 & Cocos nucifera & Indonesia & KM199372 & KM199452 & KM199529 \\
\hline & SAUCC210112 & $\begin{array}{l}\text { Ficus } \\
\text { microcarpa }\end{array}$ & China & OK149224 & OK206434 & OK206436 \\
\hline & SAUCC210113 & $\begin{array}{l}\text { Ficus } \\
\text { microcarpa }\end{array}$ & China & OK149225 & OK206435 & OK206437 \\
\hline N. protearum & CBS $114178 \mathrm{~T}$ & $\begin{array}{l}\text { Leucospermum } \\
\text { cuneiforme cv. } \\
\text { "Sunbird" }\end{array}$ & Zimbabwe & MK764278 & MK764344 & MK764322 \\
\hline
\end{tabular}




\begin{tabular}{|c|c|c|c|c|c|c|}
\hline \multirow[t]{2}{*}{ Species } & \multirow[t]{2}{*}{ Strain } & \multirow[t]{2}{*}{ Host/substrate } & \multirow[t]{2}{*}{ Country } & \multicolumn{3}{|c|}{ GenBank accession number } \\
\hline & & & & ITS & TUB2 & TEF1 \\
\hline N. rhizophorae & MFLUCC 17-1550 T & $\begin{array}{l}\text { Rhizophora } \\
\text { mucronata }\end{array}$ & Thailand & KM199360 & KM199430 & KM199524 \\
\hline \multirow[t]{2}{*}{ N. rosae } & CBS 124745 & $\begin{array}{l}\text { Paeonia } \\
\text { suffruticosa }\end{array}$ & USA & KM199359 & KM199429 & KM199523 \\
\hline & CBS $101057 \mathrm{~T}$ & Rosa sp. & $\begin{array}{l}\text { New } \\
\text { Zealand }\end{array}$ & KY885239 & KY885245 & KY885243 \\
\hline \multirow[t]{2}{*}{ N. rosicola } & CFCC 51992 T & Rosa chinensis & China & KY885239 & KY885245 & KY885243 \\
\hline & CFCC 51993 & Rosa chinensis & China & KY885240 & KY885246 & KY885244 \\
\hline N. saprophytica & CBS $115452 \mathrm{~T}$ & Magnolia sp. & China & KM199345 & KM199433 & KM199538 \\
\hline \multirow[t]{2}{*}{ N. sichuanensis } & $\begin{array}{l}\text { CFCC } 54338= \\
\text { SM15-1 T }\end{array}$ & $\begin{array}{l}\text { Castanea } \\
\text { mollissima }\end{array}$ & China & MW166231 & MW218524 & MW199750 \\
\hline & SM15-1C & $\begin{array}{l}\text { Castanea } \\
\text { mollissima }\end{array}$ & China & MW166232 & MW218525 & MW199751 \\
\hline N. sonneratae & MFLUCC 17-1745 T & $\begin{array}{l}\text { Sonneronata } \\
\text { alba }\end{array}$ & Thailand & MK764280 & MK764346 & MK764324 \\
\hline N. surinamensis & CBS $450.74 \mathrm{~T}$ & $\begin{array}{l}\text { soil under } \\
\text { Elaeis } \\
\text { guineensis }\end{array}$ & Suriname & KM199351 & KM199465 & KM199518 \\
\hline N. thailandica & MFLUCC 17-1730 T & $\begin{array}{l}\text { Rhizophora } \\
\text { mucronata }\end{array}$ & Thailand & MK764281 & MK764347 & MK764325 \\
\hline N. vitis & MFLUCC 15-1265 T & $\begin{array}{l}\text { Vitis vinifera } \\
\text { cv. "Summer } \\
\text { black" }\end{array}$ & China & KU140694 & KU140685 & KU140676 \\
\hline N. zimbabwana & CBS $111495 \mathrm{~T}$ & $\begin{array}{l}\text { Leucospermum } \\
\text { cunciforme cv. } \\
\text { "Sunbird" }\end{array}$ & Zimbabwe & $J X 556231$ & KM199456 & KM199545 \\
\hline \multirow{2}{*}{$\begin{array}{l}\text { Nonappendiculata } \\
\text { quercina }\end{array}$} & CBS $116061 \mathrm{~T}$ & Quercus suber & Italy & MH553982 & MH554641 & MH554400 \\
\hline & CBS 270.82 & $\begin{array}{l}\text { Quercus } \\
\text { pubescens }\end{array}$ & Italy & MH554025 & MH554701 & MH554459 \\
\hline $\begin{array}{l}\text { Pestalotiopsis } \\
\text { australasiae }\end{array}$ & CBS $114126 \mathrm{~T}$ & Knightia sp. & $\begin{array}{l}\text { New } \\
\text { Zealand }\end{array}$ & KM199297 & KM199409 & KM199499 \\
\hline P. australis & CBS $114193 \mathrm{~T}$ & Grevillea sp. & Australia & KM199332 & KM199383 & KM199475 \\
\hline P. grevilleae & CBS $114127 \mathrm{~T}$ & Grevillea sp. & Australia & KM199300 & KM199407 & KM199504 \\
\hline P. hollandica & CBS 265.33 T & $\begin{array}{l}\text { Sciadopitys } \\
\text { verticillata }\end{array}$ & $\begin{array}{l}\text { The } \\
\text { Netherlands }\end{array}$ & KM199328 & KM199388 & KM199481 \\
\hline P. kenyana & CBS $442.67 \mathrm{~T}$ & Coffea sp. & Kenya & KM199302 & KM199395 & KM199502 \\
\hline P. knightiae & CBS $114138 \mathrm{~T}$ & Knightia sp. & $\begin{array}{l}\text { New } \\
\text { Zealand }\end{array}$ & KM199310 & KM199408 & KM199497 \\
\hline \multirow[t]{3}{*}{ P. licualacola } & HGUP4057 T & Licuala grandis & China & KC492509 & KC481683 & KC481684 \\
\hline & SAUCC210087 & Ilex chinensis & China & OK087323 & OK104872 & OK104879 \\
\hline & SAUCC210088 & Ilex chinensis & China & OK087324 & OK104873 & OK104880 \\
\hline
\end{tabular}

Isolates marked with "T" are ex-type or ex-epitype strains. 


\begin{tabular}{|c|c|c|c|c|c|c|}
\hline \multirow[t]{2}{*}{ Species } & \multirow[t]{2}{*}{ Strain } & \multirow[t]{2}{*}{ Host/substrate } & \multirow[t]{2}{*}{ Country } & \multicolumn{3}{|c|}{ GenBank accession number } \\
\hline & & & & ITS & TUB2 & TEF1 \\
\hline P. oryzae & CBS $353.69 \mathrm{~T}$ & Oryza sativa & Denmark & KM199299 & KM199398 & KM199496 \\
\hline P. parva & CBS 278.35 & $\begin{array}{l}\text { Leucothoe } \\
\text { fontanesiana }\end{array}$ & - & KM199313 & KM199405 & KM199509 \\
\hline P. portugalica & CBS $393.48 \mathrm{~T}$ & - & Portugal & KM199335 & KM199422 & KM199510 \\
\hline $\begin{array}{l}P . \\
\text { spathuliappendiculata }\end{array}$ & CBS $144035 \mathrm{~T}$ & $\begin{array}{l}\text { Phoenix } \\
\text { canariensis }\end{array}$ & Australia & MH554172 & MH554845 & MH554607 \\
\hline $\begin{array}{l}\text { Pseudopestalotiopsis } \\
\text { cocos }\end{array}$ & CBS $272.29 \mathrm{~T}$ & Cocos nucifera & Indonesia & KM199378 & KM199467 & KM199553 \\
\hline Pse. elaeidis & CBS 413.62 T & $\begin{array}{l}\text { Elaeis } \\
\text { guineensis }\end{array}$ & Nigeria & MH554044 & MH554720 & MH554479 \\
\hline Pse. indica & CBS $459.78 \mathrm{~T}$ & Rosa sinensis & India & KM199381 & KM199470 & KM199560 \\
\hline Seiridium papillatum & CBS $340.97 \mathrm{~T}$ & $\begin{array}{l}\text { Eucalyptus } \\
\text { delegatensis }\end{array}$ & Australia & LT853102 & LT853250 & MH554468 \\
\hline Seir. phylicae & CBS $133587 \mathrm{~T}$ & Phylica arborea & $\begin{array}{l}\text { Tristan da } \\
\text { Cunha }\end{array}$ & LT853091 & LT853238 & LT853188 \\
\hline
\end{tabular}

Phylogenetic analyses

Newly generated sequences in this study were aligned with additional related sequences downloaded from GenBank (Table 1) using MAFFT 7 online service with the Auto strategy (Katoh et al. 2019, http://mafft.cbrc.jp/alignment/server/). To establish the identity of the isolates at species level, phylogenetic analyses were conducted first individually for each locus and then as combined analyses of three loci (ITS, TUB2 and TEF1- $a$ ). Phylogenetic analyses were based on maximum likelihood (ML) and Bayesian inference $(\mathrm{BI})$ for the multi-locus analyses. For BI, the best evolutionary model for each partition was determined using MrModeltest v. 2.3 (Nylander 2004) and incorporated into the analyses. ML and BI were run on the CIPRES Science Gateway portal (https://www.phylo.org/) (Miller et al. 2012) using RaxML-HPC2 on XSEDE (8.2.12) (Stamatakis 2014) and MrBayes on XSEDE (3.2.7a) (Huelsenbeck \& Ronquist 2001; Ronquist \& Huelsenbeck 2003; Ronquist et al. 2012), respectively. Four Markov chains were run for two runs from random starting trees for 5 million generations (ITS + TUB2 + TEF1- $a$ ) until the split deviation frequency value $<0.01$, and trees were sampled every 1000 generation. The first quarter generations were discarded as burn-in. A majority rule consensus tree of all remaining trees was calculated.

The resulting trees were plotted using FigTree v. 1.4.4 (http://tree.bio.ed.ac.uk/software/figtree) and edited with Adobe Illustrator CC 2019. New sequences generated in this study were deposited at GenBank (https://www.ncbi.nlm.nih.gov; Table 1).

\section{Results}

Phylogeny

Nine strains of Sporocadaceae isolated from plant hosts from Hainan, China, were grown in culture and used for analyses of molecular sequence data. The combined dataset of ITS + TUB2 + TEF1- $a$ has an aligned length of 2285 total characters (ITS: 1616, TUB2: 617-1570, TEF1- $a$ : 1571-2285) including gaps, of which 1119 characters are constant, 285 variable characters are parsimony-uninformative, and 881 are parsimony-informative. For the $B I$ and $M L$ analyses, the substitution model $S Y M+I+G$ for ITS, HKY +G for TUB2 and GTR+I+G for TEF1- $a$ were selected and incorporated into the analyses. The MCMC analysis of the three concatenated genes run for 2,650,000 generations, resulting in 2651 trees. The initial 662 trees representative of the analysis burn-in phase was discarded, while the remaining trees were used to calculate posterior probabilities in the majority rule consensus trees (Fig. 1; first value: PP $\geq 0.50$ shown). The alignment contained a total of 1453 unique site patterns (ITS: 269 , 
TUB2: 670, TEF1- $a$. 514). The ML tree topology confirmed the tree topologies obtained from the $\mathrm{BI}$ analyses, and therefore, only the ML tree is presented (Fig. 1).

ML bootstrap support values ( $\geq 50 \%$ ) and Bayesian posterior probability ( $\geq 0.50$ ) are shown as first and second position above nodes, respectively. The 81 strains were assigned to 59 species clades based on the three gene loci phylogeny (Fig. 1). Based on the multi-locus phylogeny and morphology, nine isolates were assigned to four species, including Monochaetia schimae sp. nov., Neopestalotiopsis haikouensis sp. nov., Neopestalotiopsis piceana, Pestalotiopsis licualacola, respectively.

\section{Taxonomy}

Monochaetia schimae Z.X. Zhang, X.G. Zhang \& J.W. Xia, sp. nov. Fig. 2.

\section{MycoBank 841381}

Etymology: Name refers to the genus of the host plant Schima superba.

Type: China, Hainan Province: East Harbour National Nature Reserve, on diseased leaves of Schima superba, 23 May 2021, Z.X. Zhang (holotype HSAUP212201; ex-type living culture SAUCC212201). GenBank deposition numbers of sequences derived from type: ITS, MZ577565; TUB2, OK104867; TEF1-a, OK104874.

Description: Colonies on PDA 39.0-45.0 mm in diameter after 15 days at $25^{\circ} \mathrm{C}$ in darkness, growth rate $2.5-3.0 \mathrm{~mm}$ diam/day, irregularly circular, raised, dense surface with lobate edge, zonate with different sector light brown at the margin, brown at the center; reverse brown at the margin, dark brown at the center. Sexual morph: Undetermined. Asexual morph: Leaf spots irregular, pale brown in center, brown to tan at margin. Conidiomata solitary, scattered, black, raising above surface of culture medium, subglobose, exuding black conidial droplets from central ostioles after 10 days in light at $25^{\circ} \mathrm{C}$. Conidiophores cylindrical, hyaline, smooth-walled. Conidiogenous cells 9.0-16.5 × 1.2-2.2 $\mu \mathrm{m}$, phialidic, ampulliform, discrete, hyaline, smooth, thin-walled. Conidia $18.0-24.0 \times 4.5-6.0 \mu \mathrm{m}$, mean $\pm \mathrm{SD}=20.5 \pm 1.1 \times 5.5 \pm 0.4 \mu \mathrm{m}$, fusiform, tapering at both ends, 4-septate; apical cell 2.0-4.0 $\mu \mathrm{m}$ long, conic, hyaline and smooth-walled; three median cells together 12.5-15.5 $\mu \mathrm{m}$ long, mean $\pm S D=14.2 \pm 0.7 \mu \mathrm{m}$, doliiform, brown, rough-walled, upper second cell 3.8-5.3 $\mu \mathrm{m}$ long, upper third cell 3.4-5.0 $\mu \mathrm{m}$ long, upper fourth cell 4.4-5.4 $\mu \mathrm{m}$ long; basal cell 2.2-4.5 $\mu \mathrm{m}$ long, conical, hyaline and smooth-walled; apical appendage 7.0-12.5 $\mu \mathrm{m}$ long (mean $=9.2 \mu \mathrm{m})$, single, central, tubular, filiform; basal appendage 2.5-5.0 $\mu \mathrm{m}$ long, single, tubular, filiform.

Additional specimen examined: China, Hainan Province: East Harbour National Nature Reserve, 23 May 2021, Z.X. Zhang. On diseased leaves of Schima superba (Theaceae), paratype HSAUP212202, ex-paratype culture SAUCC212202; on diseased leaves of Schima superba (Theaceae), paratype HSAUP212203, ex-paratype culture SAUCC212203.

Notes: Monochaetia schimae is introduced based on the multi-locus phylogenetic analysis, with three isolates clustering separately in a well-supported clade $(\mathrm{ML} / \mathrm{BI}=100 / 1)$. M. schimae is most closely related to $M$. ilexae (CBS 101009), but distinguished based on ITS, TUB2 and TEF1- $a$ loci by 94 nucleotide differences in the concatenated alignment, in which 18/526 are distinct in the ITS region, 32/698 in the TUB2 region and 44/462 in the TEF1-a region. Morphologically, M. schimae differ from M. ilexae in its smaller conidia (18.0-24.0 × 4.5-6.0 vs. 20.0-27.0 × 3.0-5.0 $\mu \mathrm{m})$. Therefore, we establish this fungus as a novel species.

Neopestalotiopsis haikouensis Z.X. Zhang, X.G. Zhang \& J.W. Xia, sp. nov. Fig. 3.

\section{MycoBank 841382}

Etymology: Named after the host collection, Haikou City.

Type: China, Hainan Province, Haikou City: East Harbour National Nature Reserve, on diseased leaves of Ilex chinensis. 23 May 2021, Z.X. Zhang (holotype HSAUP212271; ex-type living culture SAUCC212271). GenBank deposition numbers of sequences derived from type: ITS, OK087294; TUB2, OK104870; TEF1-a, OK104877. 
Description: Colonies on PDA incubated at $25^{\circ} \mathrm{C}$ in the dark with an average radial growth rate of $7.0-9.0 \mathrm{~mm} /$ day and occupying an entire $90 \mathrm{~mm}$ Petri dish in $7 \mathrm{~d}$; edge undulate, white to grey white, with moderate aerial mycelium on the surface, with black, gregarious conidiomata; reverse similar in colour. Sexual morph: Undetermined. Asexual morph: Leaf spots irregular, grey white in center, brown to tan at margin. Conidiomata globose to clavate, solitary or confluent, embedded or semi-immersed to erumpent, dark brown, exuding globose, dark brown to black conidial masses. Conidiophores indistinct, often reduced to conidiogenous cells. Conidiogenous cells discrete, subcylindrical to ampulliform, hyaline, 5.0-10.0 × 2.0-6.0 $\mu \mathrm{m}$, apex 1.0-2.0 $\mu \mathrm{m}$ diam. Conidia fusoid, ellipsoid, straight to slightly curved, 4-septate, 16.0-22.0 $\times 4.5-7.0 \mu \mathrm{m}$, mean $\pm \mathrm{SD}=20.0 \pm 1.8 \times 5.5 \pm 0.4 \mu \mathrm{m}$; basal cell conic with a truncate base, hyaline, rugose and thin-walled, 3.0-4.5 $\mu \mathrm{m}$ long; three median cells doliiform, $11.5-15.0 \mu \mathrm{m}$ long, mean $\pm \mathrm{SD}=13.2 \pm 1.0 \mu \mathrm{m}$, wall rugose, versicoloured, septa darker than the rest of the cell, second cell from the base pale brown, 3.5-5.5 $\mu \mathrm{m}$ long; third cell honey-brown, 4.0-6.0 $\mu \mathrm{m}$ long; fourth cell brown, 3.8-5.7 $\mu \mathrm{m}$ long; apical cell 2.5-5.5 $\mu \mathrm{m}$ long, hyaline, cylindrical to subcylindrical, thin- and smooth-walled; with 2-3 tubular apical appendages (mostly 3), arising from the apical crest, unbranched, filiform, 13.5-24.0 $\mu \mathrm{m}$ long, mean $\pm \mathrm{SD}=19.1 \pm 3.5 \mu \mathrm{m}$; basal appendage 2.0-7.0 $\mu \mathrm{m}$ long, single, tubular, unbranched, centric.

Additional specimen examined: China, Hainan Province: East Harbour National Nature Reserve, 23 May 2021, Z.X. Zhang. On diseased leaves of Ilex chinensis, paratype HSAUP212272, ex-paratype culture SAUCC212272.

Notes: Phylogenetic analysis of a combined three gene showed that Neopestalotiopsis haikouensis formed an independent clade (Fig. 1) and is phylogenetically distinct from N. aotearoa (CBS 367.54), N. piceana (CBS 254.32), N. brachiate (MFLUCC 17-1555), N. phangngaensis (MFLUCC 18-0119), N. rhizophorae (MFLUCC 17-1550), N. petila (MFLUCC 17-1738) and N. rosicola (CFCC 51993). N. haikouensis can be distinguished from the phylogenetically most closely related species $N$. aotearoa by shorter and wider conidia (16.0-22.0 × 4.5-7.0 vs. 20.0-27.0 × 3.0-5.0 $\mu \mathrm{m})$, and several loci (7/532 in the ITS region, 4/771 TUB2 and 6/487 TEF1- $a$ ). Therefore, we establish this fungus as a novel species.

Neopestalotiopsis piceana Maharachch., K.D. Hyde \& Crous, Studies in Mycology 79:146. (2014) Fig. 4.

Description: Colonies on PDA reaching 70.0-80.0 mm diam after $7 \mathrm{~d}$ at $25^{\circ} \mathrm{C}$, edge entire, whitish to pale honeycoloured, with sparse aerial mycelium on the surface, with black, gregarious conidiomata; reverse similar in colour. Sexual morph: Undetermined. Asexual morph: Leaf spots irregular, pale brown in center, brown to tan at margin. Conidiomata (on PDA) pycnidial, globose to clavate, solitary, semi-immersed, brown to black; exuding globose, dark brown to black conidial masses. Conidiophores reduced to conidiogenous cells. Conidiogenous cells discrete, ampulliform to lageniform, hyaline, smooth and thin walled, simple, $4.0-12.0 \times$

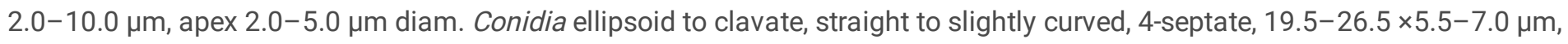
mean $\pm S D=22.7 \pm 0.8 \times 6.1 \pm 0.4 \mu \mathrm{m}$; somewhat constricted at septa; basal cell obconic with truncate base, rugose and thinwalled, 2.7-5.0 $\mu \mathrm{m}$ long; three median cells 12.0-16.0 $\mu \mathrm{m}$ long, mean $\pm \mathrm{SD}=14.7 \pm 0.9 \mu \mathrm{m}$, doliiform, verruculose, versicoloured, septa darker than the rest of the cell second cell from base pale brown, 4.0-5.7 $\mu \mathrm{m}$ long; third cell dark brown, 3.5-5.2 $\mu \mathrm{m}$ long; fourth cell brown, 3.8-5.8 $\mu \mathrm{m}$ long; apical cell obconic, hyaline, thin and smooth-walled, 2.5-5.2 $\mu \mathrm{m}$ long; with 3 tubular apical appendages, arising from the apical crest, flexuous, unbranched, $21.0-32.0 \mu \mathrm{m}$ long, mean $\pm \mathrm{SD}=24.8 \pm 3.5 \mu \mathrm{m}$; basal appendage single, tubular, unbranched, centric, 2.7-6.5 $\mu \mathrm{m}$ long.

Specimens examined: China, Hainan Province: Five Fingers Group Scenic Area, 20 May 2021, Z.X. Zhang. On diseased leaves of Ficus microcarpa, paratype HSAUP210112, ex-paratype culture SAUCC210112; on diseased leaves of Ficus microcarpa, paratype HSAUP210113, ex-paratype culture SAUCC210113.

Notes: In the present study, two strains (SAUCC210112 and SAUCC210113) from symptomatic leaves of Ficus microcarpa were similar to Neopestalotiopsis piceana (CBS 394.48) (Maharachch. et al. 2014) based on phylogeny (Fig. 1). Morphologically, our strains were similar to N. piceana, which was originally described with an asexual morph on wood of Picea sp., Cocos nucifera and fruit of Mangifera indica in China, but the sexual morph of $N$. piceana was undetermined. We therefore identify our strains as N. piceana.

Pestalotiopsis licualacola K. Geng, Y. Song, K.D. Hyde \& Yong Wang bis, Phytotaxa 88 (3):51. (2013) Fig. 5. 
Description: Colonies on PDA incubated at $25^{\circ} \mathrm{C}$ in the dark with an average radial growth rate of $7.0-9.0 \mathrm{~mm} /$ day and occupying an entire $90 \mathrm{~mm}$ Petri dish in $7 \mathrm{~d}$, with edge undulate, whitish, aerial mycelium on surface, fruiting bodies black, concentric; reverse of culture yellow to pale brown. Sexual morph: Undetermined. Asexual morph: Leaf spots irregular, pale brown in center, brown to tan at margin. Conidiomata (pycnothyria) solitary, scattered, black, raising above surface of culture medium, subglobose. Conidiophores cylindrical, hyaline, smooth-walled. Conidiophores most often indistinct. Conidiogenous cells discrete, hyaline, simple, filiform, 5.5-10.0 $\mu \mathrm{m}$ long. Conidia 18.0-24.5 × 4.0-5.5 $\mu \mathrm{m}$, mean \pm SD $=20.5 \pm 1.9 \times 5.3 \pm 0.3 \mu \mathrm{m}$, fusiform, straight to slightly curved, 4-septate, smooth, greyish brown; basal cell conical, hyaline, thin-walled, $2.8-6 \mu \mathrm{m}$ long; with three median cells, dark brown, concolorous, septa and periclinal walls darker than the rest of the cell, together 11.5-16.0 $\mu \mathrm{m}$ long, mean $\pm \mathrm{SD}=13.2 \pm 1.2 \mu \mathrm{m}$; second cell from base 3.4-5.5 $\mu \mathrm{m}$; third cell 3.3-4.7 $\mu \mathrm{m}$; fourth cell 3.5-5.1 $\mu \mathrm{m}$; apical cell hyaline, conic to subcylindrical, 3.1-5.3 $\mu \mathrm{m}$; with 1-3 tubular apical appendages (mostly 1 ) without knobs, arising from the apex of the apical cell, 10.0-20.5 $\mu \mathrm{m}$ long, mean $\pm \mathrm{SD}=16.0 \pm 4.0 \mu \mathrm{m}$; basal appendage filiform, short.

Specimens examined: China, Hainan Province: East Harbour National Nature Reserve, 23 May 2021, Z.X. Zhang. On diseased leaves of Ilex chinensis, paratype HSAUP210087, ex-paratype culture SAUCC210087; on diseased leaves of Ilex chinensis, paratype HSAUP210088, ex-paratype culture SAUCC210088.

Notes: In the present study, two strains (SAUCC210087 and SAUCC210088) from symptomatic leaves of Ilex chinensis were similar to Pestalotiopsis licualacola (HGUP4057) (Geng et al. 2013) based on phylogeny (Fig. 1). Morphologically, our strains were similar to $P$. licualacola, which was originally described with an asexual morph on leaves of Licuala grandis in China, but the sexual morph of $P$. licualacola was undetermined. We therefore identify our strains as $P$. licualacola.

\section{Discussion}

In the study of its phylogenetic affinity and position in the Ascomycota hierarchical system, Liu et al. (2019) placed a series of coelomycetous fungi with appendage-bearing conidia in the newly introduced family Sporocadaceae (type genus: Sporocadus Corda). In most genera, conidiomata pycnidial, acervular or stromatic, semi-immersed or immersed, scattered, gregarious or confluent, glabrous; conidiophores branched or reduced to conidiogenous cells, mostly hyaline, smooth; conidiogenous cells ampulliform, lageniform, cylindrical or subcylindrical, hyaline, sometimes pale brown; conidia septate, smooth, undulate or verruculose, fusoid, subcylindrical or cylindrical, straight or curved; end cells mostly hyaline, or sometimes pale brown; median cells pale brown to dark brown, or sometime almost colourless; appendages on the end cells present, or absent in some genera, if present, tubular, filiform, straight or flexuous, attenuated or not, branched or unbranched.

In the previous study, Liu et al. (2019) revealed three major clades based on the ITS/ LSU/ RPB2 phylogeny, corresponding to the three previously proposed families "Bartaliniaceae, Discosiaceae and Pestalotiopsidaceae". Because these family names were considered synonyms of Sporocadaceae by Jaklitsch et al. (2016), these groups are referred to as Clade 1 (Discosiaceae), Clade 2 (Pestalotiopsidaceae) and Clade 3 (Bartaliniaceae) for convenience. We picked Clade 2 of the family Sporocadaceae in this study, including Pestalotiopsis Steyaert, Neopestalotiopsis Maharachch. et al., Pseudopestalotiopsis Maharachch. et al., Monochaetia (Sacc.) Allesch., Ciliochorella Syd., Seiridium Nees and Nonappendiculata Liu et al. (Liu et al. 2019). Based on ITS/TUB2/ TEF1-a molecular data, phylogenetic analyses revealed that the retrieved three isolates (SAUCC212201, SAUCC212202 and SAUCC212203) are include in Monochaetia, four isolates (SAUCC212271, SAUCC212272, SAUCC210112 and SAUCC210113) are include in Neopestalotiopsis and two isolates (SAUCC210087 and SAUCC210088) are include in Pestalotiopsis. Owing to different nucleotides in the concatenated alignment and morphology, three isolates (SAUCC212201, SAUCC212202 and SAUCC212203) of Monochaetia were delimited as a new species, namely M. schimae and two isolates (SAUCC212271 and SAUCC212272) of Neopestalotiopsis were delimited as a new species, namely N. haikouensis. Besides, two isolates (SAUCC210112 and SAUCC210113) of Neopestalotiopsis clustered in N. piceana and two isolates (SAUCC210087 and SAUCC210088) of Pestalotiopsis clustered in P. licualacola.

In addition, members of Sporocadaceae are of particular interest with regard to the production of secondary metabolites, e.g. Pestalotiopsis, Bartalinia and Morinia (Collado et al. 2006; Gangadevi \& Muthumary 2008; Liu et al. 2009). Pestalotiopsis fici was shown to possess a very high number of gene clusters involved in bioactive compound synthesis (Wang et al. 2016). Because 
genera in this family of fungi share the same evolutionary history, it is important to found novel species and screen for novel metabolites in future studies.

\section{Declarations}

Acknowledgments This work was supported by the National Natural Science Foundation of China (no. 31900014, U2002203).

Author contribution Conceived and designed the experiments: JW Xia. Performed the experiments: ZX Zhang. Analyzed the data: TC Mu, SB Liu and RY Liu. Wrote the paper: ZX Zhang, XG Zhang and JW Xia. All authors read and approved the final manuscript.

Data availability The data, including the sequences on GenBank and specimen data on MycoBank will be available to any researcher wishing to use them for non-commercial purposes, without breaching participant confidentiality.

Declarations Conflict of interest The authors declare no competing interests.

\section{References}

1. Barr ME (1990) Prodromus to nonlichenized, pyrenomycetous members of the class Hymenoascomycetes. Mycotaxon 39:43-184

2. Barber PA, Crous PW, Groenewald JZ, Pascoe IG, Keane P (2011) Reassessing Vermisporium (Amphisphaeriaceae), a genus of foliar pathogens of eucalypts. Persoonia 27:90-118. https://doi.org/10.3767/003158511X617381

3. Carbone I, Kohn LM (1999) A method for designing primer sets for speciation studies in filamentous Ascomycetes. Mycologia 91(3):553-556. https://doi.org/10.1080/00275514.1999.12061051

4. Collado J, Platas G, Bills GF, Basilio Á, Vicente F, Rubén Tormo J, Hernández P, Teresa Díez M, Peláez F (2006) Studies on Morinia: Recognition of Morinia longiappendiculata sp. nov. as a new endophytic fungus, and a new circumscription of Morinia pestalozzioides. Mycologia 98:616-627. https://doi.org/10.1080/15572536.2006.11832665

5. Crous PW, Carris LM, Giraldo A, Groenewald JZ, Hawksworth DL, Hemández-Restrepo M, Jaklitsch WM, Lebrun MH, Schumacher RK, Stielow JB, van der Linde EJ, Vilcāne J, Voglmayr H, Wood AR (2015) The genera of fungi - fixing the application of the type species of generic names - G 2: Allantophomopsis, Latorua, Macrodiplodiopsis, Macrohilum, Milospium, Protostegia, Pyricularia, Robillarda, Rotula, Septoriella, Torula, and Wojnowicia. IMA Fungus 6:163-198. https://doi.org/10.5598/imafungus.2015.06.01.11

6. Eriksson OE, Hawksworth DL (1986) Notes on ascomycete systematics. Nos. 1-224. Systema Ascomycetum 5:113-174

7. Eriksson OE, Hawksworth DL (1987) Notes on ascomycete systematics. Nos. 464-551. Systema Ascomycetum 6:237-258

8. Gangadevi V, Muthumary J (2008) Taxol, an anticancer drug produced by an endophytic fungus Bartalinia robillardoides Tassi, isolated from a medicinal plant, Aegle marmelos Correa ex Roxb. World Journal of Microbiology Biotechnology 24:717-724. https://doi.org/10.1007/s11274-007-9530-4

9. Gao YH, Sun W, Su YY, Cai L (2014) Three new species of Phomopsis in Gutianshan Nature Reserve in China. Mycological Progress 13(1):111-121. https://doi.org/10.1007/s11557-013-0898-2

10. Glass NL, Donaldson GC (1995) Development of primer sets designed for use with the PCR to amplify conserved genes from filamentous ascomycetes. Appl Environ Microbiol 61(4):1323-1330. https://doi.org/10.1128/AEM.61.4.1323-1330.1995

11. Guo LD, Hyde KD, Liew ECY (2000) Identification of endophytic fungi from Livistona chinensis based on morphology and rDNA sequences. New Phytol 147(3):617-630. https://doi.org/10.1046/j.1469-8137.2000.00716.x

12. Hawksworth DL, Kirk PM, Sutton BC, Pegler DN (1995) Ainsworth \& Bisby's Dictionary of the Fungi, 8th edn. CAB International, Wallingford

13. Huelsenbeck JP, Ronquist F (2001) MRBAYES: Bayesian inference of phylogeny. Bioinformatics 17(17):754-755. https://doi.org/10.1093/bioinformatics/17.8.754

14. Jaklitsch WM, Gardiennet A, Voglmayr H (2016) Resolution of morphology-based taxonomic delusions: Acrocordiella, Basiseptospora, Blogiascospora, Clypeosphaeria, Hymenopleella, Lepteutypa, Pseudapiospora, Requienella, Seiridium and 
Strickeria. Persoonia 37:82-105. https://doi.org/10.3767/003158516X690475

15. Jeewon R, Liew ECY, Hyde KD (2002) Phylogenetic relationships of Pestalotiopsis and allied genera inferred from ribosomal DNA sequences and morphological characters. Mol Phylogenet Evol 25:378-392. https://doi.org/10.1016/S10557903(02)00422-0

16. Katoh K, Rozewicki J, Yamada KD (2019) MAFFT online service: multiple sequence alignment, interactive sequence choice and visualization. Brief Bioinform 20:1160-1166. https://doi.org/10.1093/bib/bbx108

17. Kumar S, Stecher G, Tamura K (2016) MEGA7: Molecular Evolutionary Genetics Analysis Version 7.0 for Bigger Datasets. Mol Biol Evol 33(7):1870-1874. https://doi.org/10.1093/molbev/msw054

18. Liu F, Bonthond G, Groenewald JZ, Cai L, Crous PW (2019) Sporocadaceae, a family of coelomycetous fungi with appendagebearing conidia. Stud Mycol 92:287-415. https://doi.org/10.1016/j.simyco.2018.11.001

19. Liu L, Li Y, Liu SC, Zheng ZH, Chen XL, Zhang H, Guo LD, Che YS (2009) Chloropestolide A, an antitumor metabolite with an unprecedented spiroketal skeleton from Pestalotiopsis fici. Org Lett 11:2836-2839. https://doi.org/10.1021/ol901039m

20. Miller MA, Pfeiffer W, Schwartz T (2012) The CIPRES science gateway: enabling high-impact science for phylogenetics researchers with limited resources. In: Proceedings of the 1st Conference of the Extreme Science and Engineering Discovery Environment. Bridging from the extreme to the campus and beyond. Association for Computing Machinery, USA, 1-8. https://doi.org/10.1145/2335755.2335836

21. Nag Raj TR (1993) Coelomycetous anamorphs with appendage-bearing conidia. Mycologue publications, Canada

22. Nylander JAA (2004) MrModelTest v. 2. Program distributed by the author. Evolutionary Biology Centre, Uppsala University

23. O’Donnell K, Cigelnik E (1997) Two divergent intragenomic rDNA ITS2 types within a monophyletic lineage of the fungus Fusarium are nonorthologous. Mol Phylogenet Evol 7:103-116. https://doi.org/10.1006/mpev.1996.0376

24. O’Donnell K, Kistler HC, Cigelnik E, Ploetz RC (1998) Multiple Evolutionary Origins of the Fungus Causing Panama Disease of Banana: Concordant Evidence from Nuclear and Mitochondrial Gene Genealogies. Proc Natl Acad Sci USA 95(5):2044-2049. https://doi.org/10.1073/pnas.95.5.2044

25. Ronquist F, Huelsenbeck JP (2003) MrBayes 3: Bayesian Phylogenetic Inference under Mixed Models. Bioinformatics 19(12):1572-1574. https://doi.org/10.1093/bioinformatics/btg180

26. Ronquist F, Teslenko M, van der Mark P, Ayres DL, Darling A, Höhna S, Larget B, Liu L, Suchard MA, Huelsenbeck JP (2012) MrBayes 3.2: efficient Bayesian phylogenetic inference and model choice across a large model space. Syst Biol 61(3):539542. https://doi.org/10.1093/sysbio/sys029

27. Samuels GJ, Müller E, Petrini O (1987) Studies in the Amphisphaeriaceae (sensu lato) 3. New species of Monographella and Pestalosphaeria and two new genera. Mycotaxon 28:473-499

28. Senanayake IC, Maharachchikumbura SSN, Hyde KD, Bhat JD, Gareth Jones EB, McKenzie EHC, Dai DQ, Daranagama DA, Dayarathne MC, Goonasekara ID, Konta S, Li WJ, Shang QJ, Stadler M, Wijayawardene NN, Xiao YP, Norphanphoun C, Li Q, Liu XZ, Bahkali AH, Kang JC, Wang Y, Wen TC, Wendt L, Xu JC, Camporesi E (2015) Towards unraveling relationships in Xylariomycetidae (Sordariomycetes). Fungal Divers 73:73-144. https://doi.org/10.1007/s13225-015-0340-y

29. Tanaka K, Endo M, Hirayama K, Okane I, Hosoya T, Sato T (2011) Phylogeny of Discosia and Seimatosporium, and introduction of Adisciso and Immersidiscosia genera nova. Persoonia 26:85-98.

https://doi.org/10.3767/003158511X576666

30. Wang B, Zhang ZW, Guo LD, Liu L (2016) New cytotoxic meroterpenoids from the plant endophytic fungus Pestalotiopsis fici. Helv Chim Acta 99:151-156. https://doi.org/10.1002/hlca.201500197

31. White TJ, Bruns T, Lee S (1990) Amplification and direct sequencing of fungal ribosomal RNA genes for phylogenetics. In: Innis MA, Gelfand DH, Sninsky JJ (eds) PCR protocols: a guide to methods and applications. Academic Press Inc, New York, pp 315-322. https://doi.org/10.1016/B978-0-12-372180-8.50042-1

32. Wijayawardene NN, Hyde KD, Wanasinghe DN, Papizadeh M, Goonasekara ID, Camporesi E, Jayarama Bhat D, McKenzie EHC, Phillips AJL, Diederich P, Tanaka K, Li WJ, Tangthirasunun N, Phookamsak R, Dai DQ, Dissanayake AJ, Weerakoon G, Maharachchikumbura SSN, Hashimoto A, Matsumura M, Bahkali AH, Wang Y (2016) Taxonomy and phylogeny of dematiaceous coelomycetes. Fungal Divers 77:1-316. https://doi.org/10.1007/s13225-016-0360-2

Page $12 / 17$ 


\section{Figures}

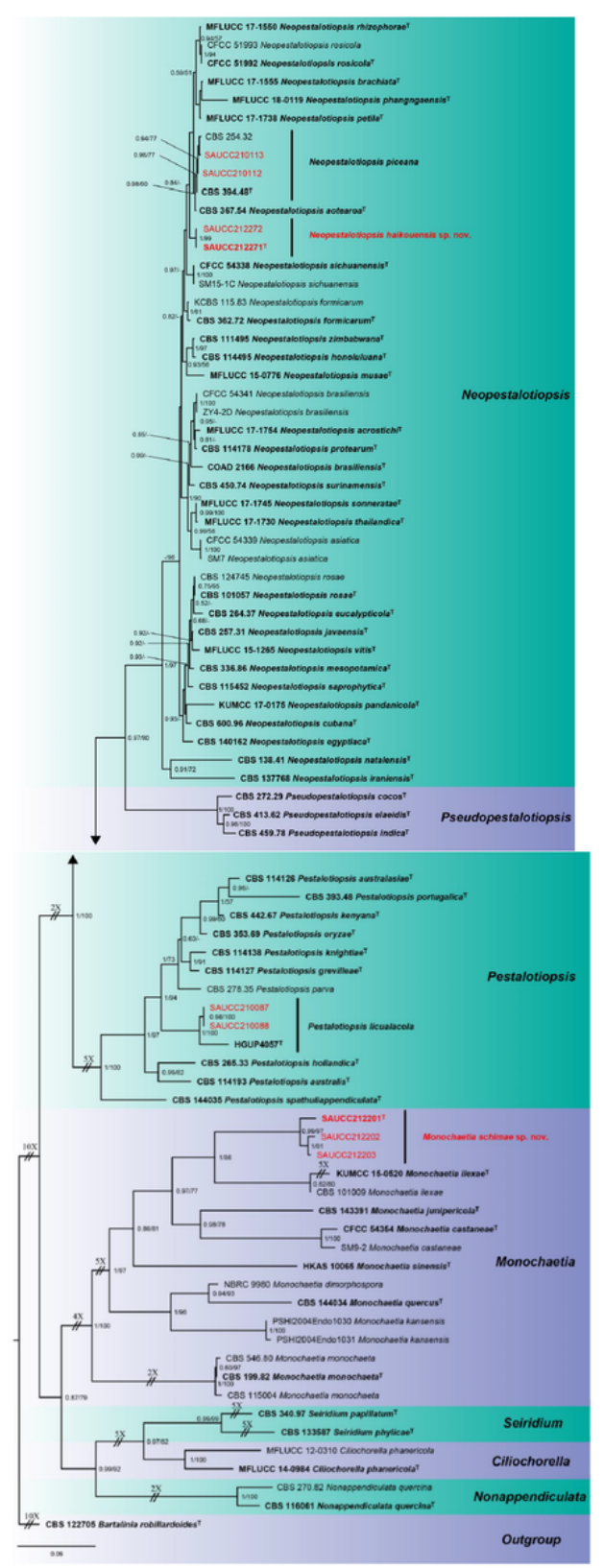

\section{Figure 1}

Phylogram of Sporocadaceae based on combined ITS, TUB2 and TEF1- $a$ genes. The BI and ML bootstrap support values above 0.50 BYPP and $50 \%$ are shown at the first and second position, respectively. The tree is rooted to Bartalinia robillardoides (CBS 122705), ex-type or ex-epitype cultures are indicated in bold face. Strains from the current study are in red. Some branches were shortened to fit them to the page - these are indicated by two diagonal lines with the number of times a branch was shortened indicated next to the lines. 


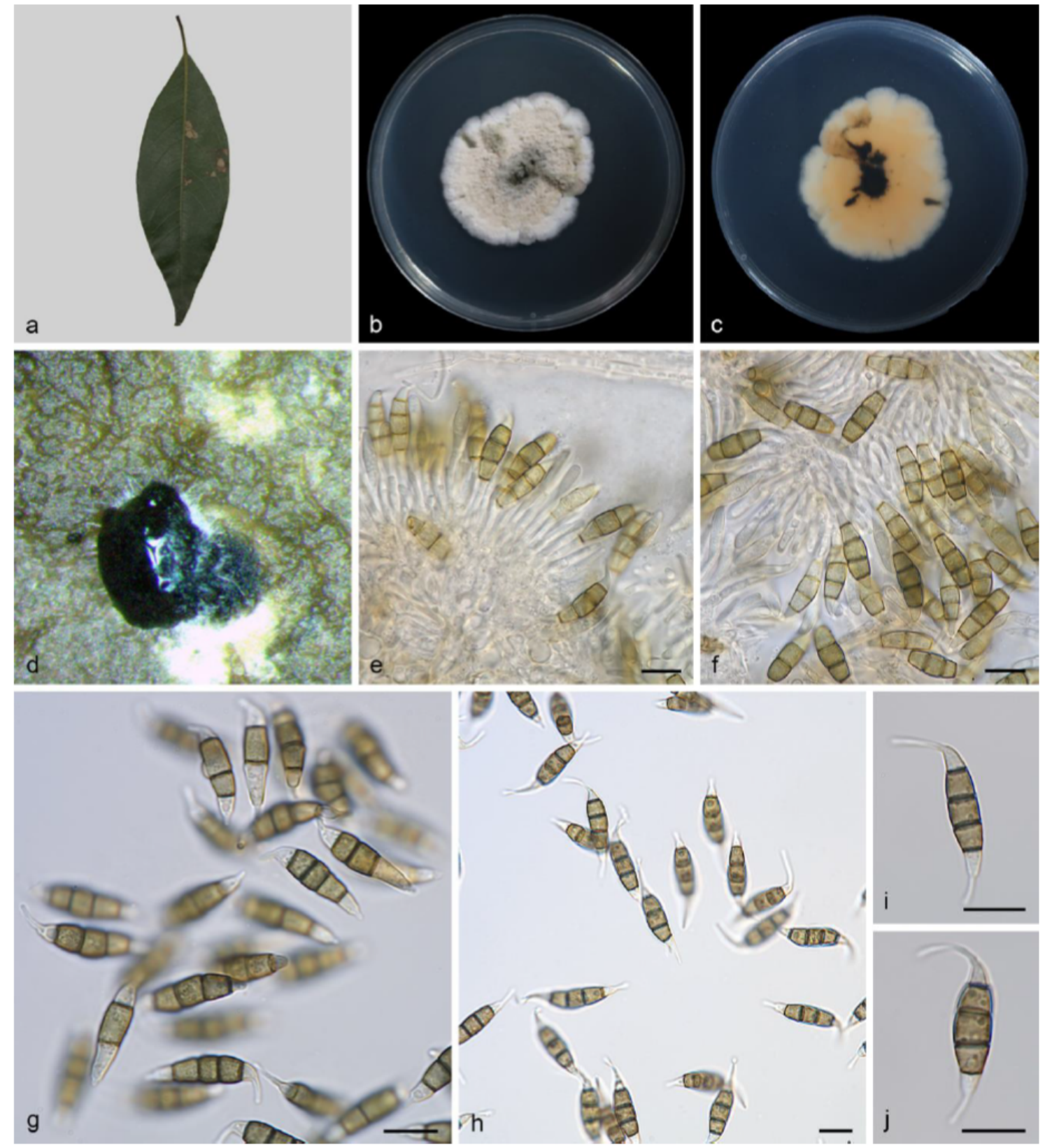

\section{Figure 2}

Monochaetia schimae (SAUCC212201). a diseased leaf of Schima superba b surface of colony after 15 days on PDA c reverse of colony after 15 days on PDA d conidiomata e, f conidiogenous cells with conidia $g-j$ conidia. Scale bars: $10 \mu m(e-j)$. 


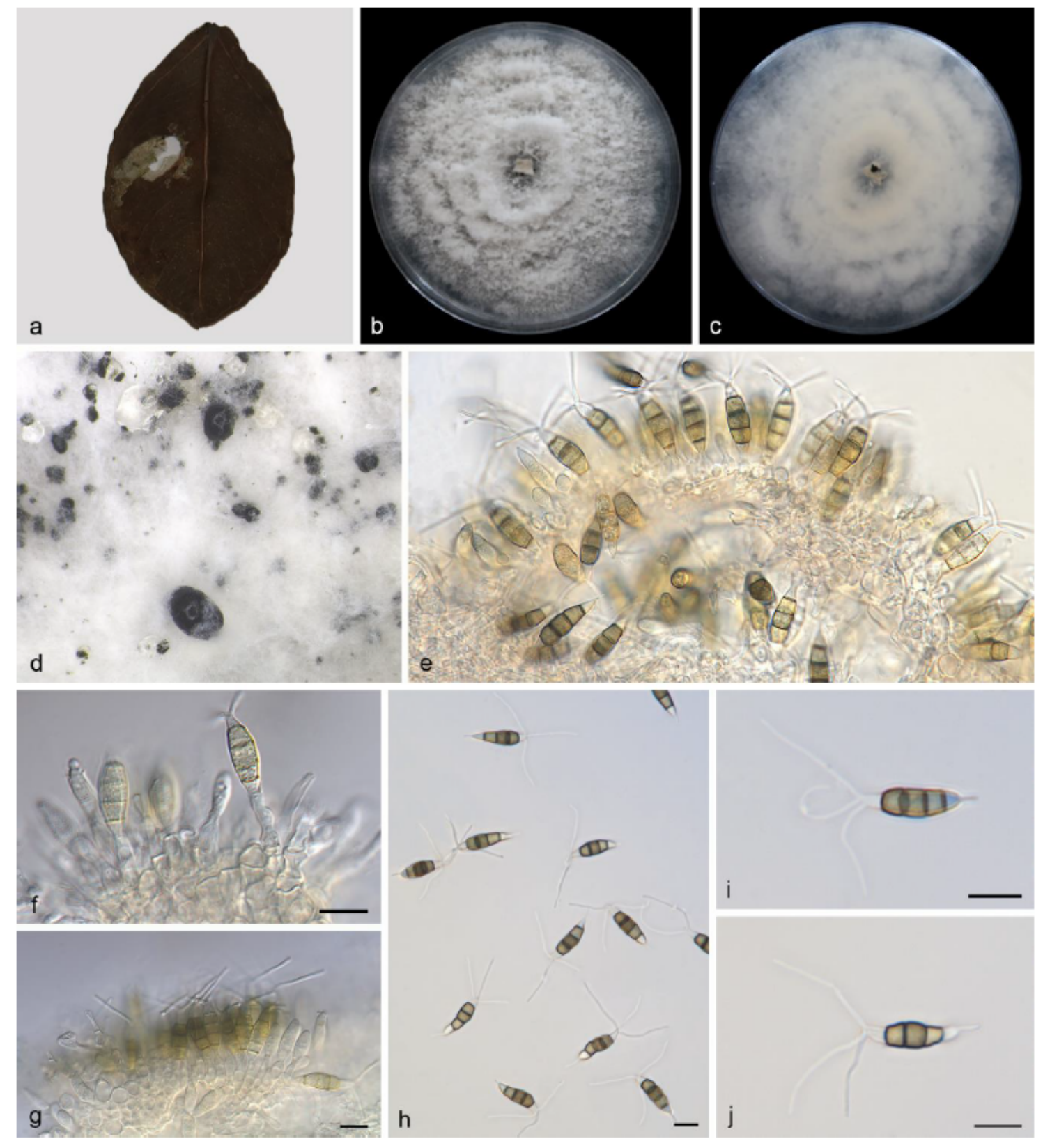

\section{Figure 3}

Neopestalotiopsis haikouensis (SAUCC212271). a diseased leaf of llex chinensis b surface of colony after 7 days on PDA c reverse of colony after 7 days on PDA d conidiomata $\mathrm{e}-\mathrm{g}$ conidiogenous cells with conidia $\mathrm{h}-\mathrm{j}$ conidia. Scale bars: $10 \mu \mathrm{m}(\mathrm{e}-\mathrm{j})$. 


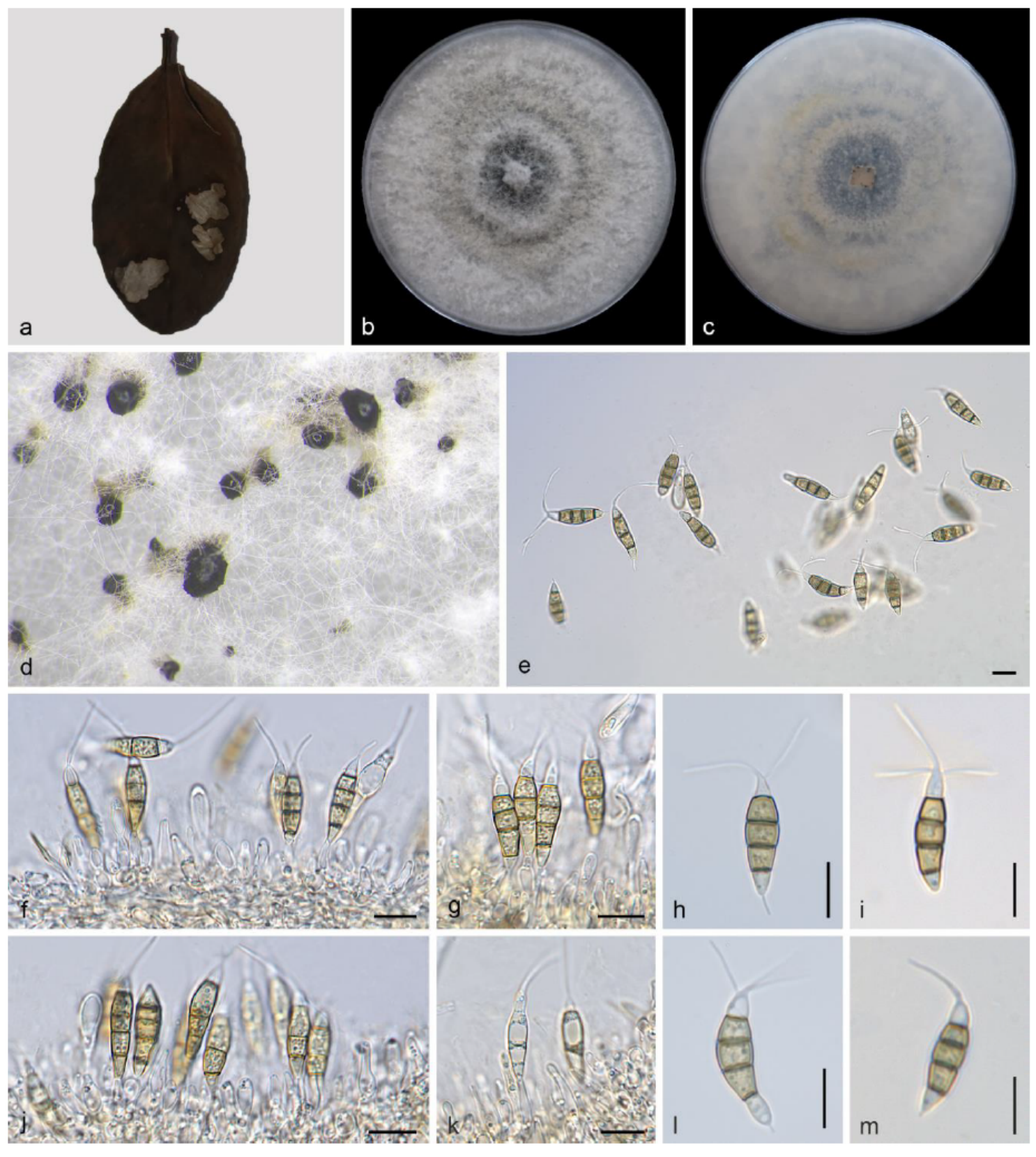

\section{Figure 4}

Neopestalotiopsis piceana (SAUCC210112). a diseased leaf of Ficus microcarpa b surface of colony after 7 days on PDA c reverse of colony after 7 days on PDA d conidiomata f, $\mathrm{g}, \mathrm{j}, \mathrm{k}$ conidiogenous cells with conidia e, h, i, I, m conidia. Scale bars: 10 $\mu \mathrm{m}(\mathrm{e}-\mathrm{m})$. 


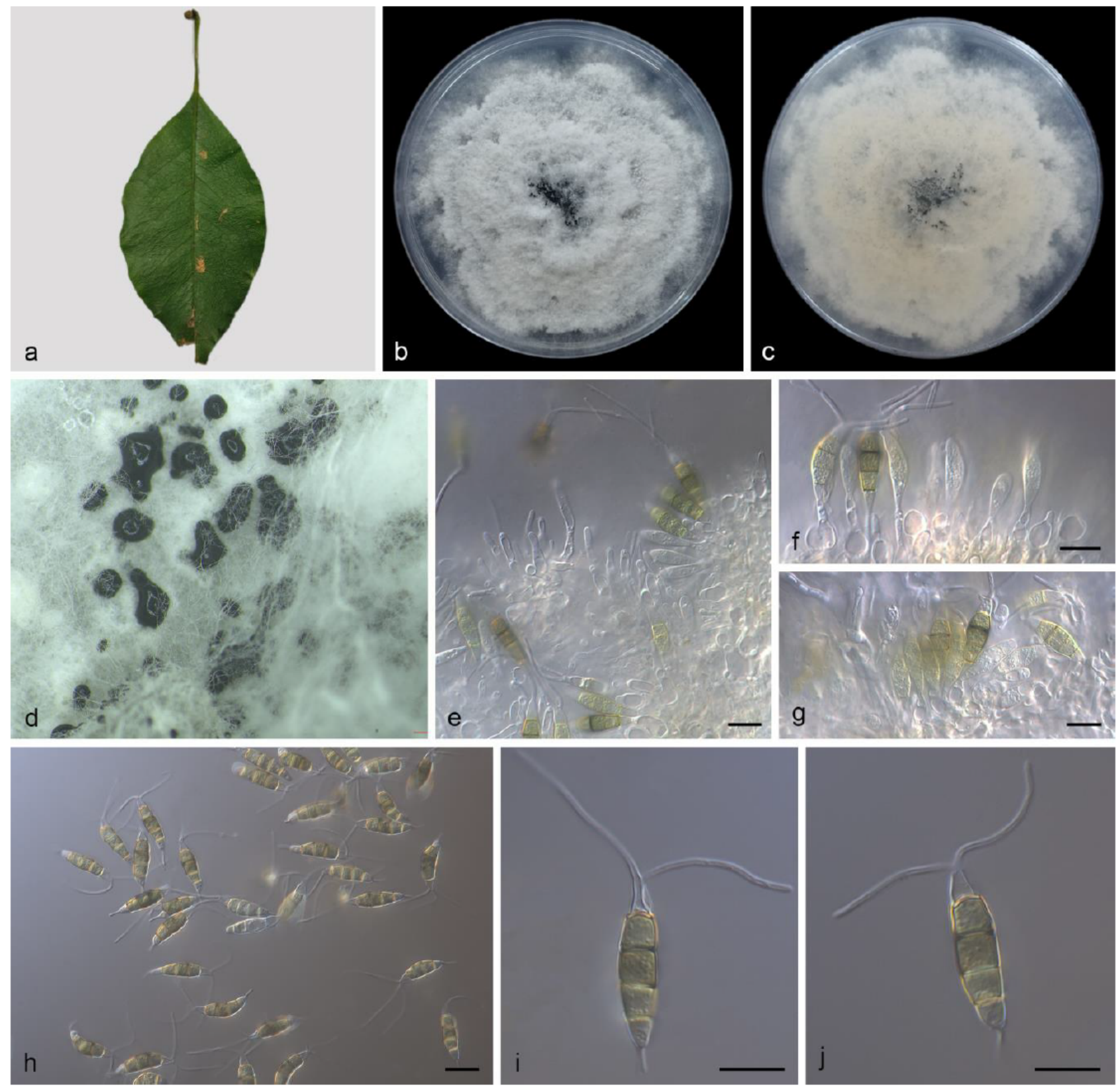

\section{Figure 5}

Pestalotiopsis licualacola (SAUCC210087). a diseased leaf of llex chinensis b surface of colony after 7 days on PDA c reverse of colony after 7 days on PDA d conidiomata $\mathrm{e}-\mathrm{g}$ conidiogenous cells with conidia $\mathrm{h}-\mathrm{j}$ conidia. Scale bars: $10 \mu \mathrm{m}(\mathrm{e}-\mathrm{j})$. 